\title{
DIE PROPORTIO QUINTUPLA IN EINIGEN VILLANCICOS DES CANCIONERO MUSICAL DE PALACIO
}

Unter den Villancicos des Cancionero musical de Palacio, der bedeutendsten Quelle weltlicher spanischer Musik aus der Zeit um 1500, fallen sieben Stücke auf, die in der seltenen Proportio quintupla notiert sind. Es handelt sich dabei um folgende Liedkompositionen:

\begin{tabular}{|c|c|c|c|c|c|c|}
\hline & Komponist & 1. Vers & Stimmenzahl & Mensurzeichen & Barbieri & Anglés \\
\hline 1 & Escobar & Las mis penas, madre & 4 & $\begin{array}{l}5 \\
1\end{array}$ & 48 & 59 \\
\hline 2 & Encina & Amor con fortuna & 4 & $\begin{array}{ll}C & 5 \\
& 1\end{array}$ & 79 & 102 \\
\hline 3 & anonym & Pensad ora'n al & 3 & $\begin{array}{ll}C & 5 \\
& 1\end{array}$ & 102 & 151 \\
\hline 4 & Anchieta & Dos ánades, madre & 3 & $\begin{array}{ll}0 & 5 \\
& 1\end{array}$ & 115 & 177 \\
\hline 5 & Fernandes & De ser mal casada & 4 & $\begin{array}{l}5 \\
1\end{array}$ & 132 & 197 \\
\hline 6 & Anchieta & Con amores, mi madre & 4 & $\begin{array}{ll}0 & 5 \\
& 1\end{array}$ & 215 & 335 \\
\hline 7 & Encina & Tan buen ganadico & 4 & 32 & 393 & 426 \\
\hline
\end{tabular}

Das Mensurzeichen der Proportio quintupla, das hier nicht innerhalb eines Satzes eine Veränderung des Zeitmaßes anzeigt, in welcher Konstellation man eine Fünfergruppierung in der Musik dieser Zeit manchmal antreffen kann', sondern als Vorzeichnung

1. S. a. Willi Apel: Die Notation der polyphonen Musik 900 - 1600. S. $170 \mathrm{ff}$.

Herrn Dr. Erich Tremmel von der Universität Augsburg verdanke ich einen Hinweis auf die Regola seconda in Sylvestro Ganassis Lehrwerk La Fontegara aus dem Jahr 1535. In einem sehr umfangreichen Kapitel stellt Ganassi hierin die Diminutionen in der sog. Proportio sesquiquarta dar, welche eine Unterteilung in fünf gleiche Werte, $d$. $h$. die Proportion 5/4 verlangen. Aber auch hier handelt es sich um kein durchgängiges Metrum, sondern um eine Form der Verzierung, die nur punktuell eingesetzt wird und ihren Reiz gerade dadurch erhallt, daß sie von ihrer Umgebung rhythmisch abweicht. 
vor einem ganzen Lied, mutete die beiden Herausgeber des Cancionero, Barbieri und Anglés, wohl derart seltsam an, daß sie es nicht bzw. kaum wagten, die Stücke im 5Metrum zu lesen. Barbieri wählt für alle Lieder ein 6-Metrum (6/2), Anglés schreibt bei den ersten drei Villancicos 12/8, beim vierten 3/4 und beim fünften $6 / 8$ vor. Erst bei den letzten beiden Stücken entschließt er sich, die Ziffern 5 und 1 bzw. 3 und 2 als Proportionsangaben zur Unterteilung des integer valor zu lesen, und überträgt einmal im $5 / 4$ und einmal im $5 / 8$.

\section{Notenbeispiel 1: Villancicos 6 und 7 in der Übertragung von Anglés}

Im Kommentar zu diesen beiden letztgenannten Kompositionen räumt er ein, daß auch die übrigen wohl besser im 5-Metrum übertragen worden wären:

Nr. 335: Fuentes: Ma. Transcribimos la "proportio quintupla" en $5 / 4(=5 / 8)$, compás que aparece en el zortzico vasco y en otras muchas canciones de las diversas regiones de España. Los números 59,102,151 y 197 del tomo 1 del presente Cancionero contarían acaso mejor con ese compás.

Nr. 426: Fuentes: Ma. Transcribimos el compás 32 del manuscrito como en $5 / 8$ puesto que el carácter de la pieza exige este ritmo.
Nr. 335: Quelle: Ma. Wir haben die "Proportio quintupla" im 5/4 (=5/8) übertragen, einer Taktart, die im baskischen Zortcico erscheint und in vielen anderen Liedern aus verschiedenen Gebieten Spaniens. Die Nummern 59, 102, 151 und 197 im ersten Band des verliegenden Cancionero wären wohl auch besser in dieser Taktart übertragen.

Nr. 426: Quelle: Ma. Wir haben die Taktart 32 des Manuskripts als 5/8 übertragen, da der Charakter des Stückes dies verlangt.

Da Anglés nur die letzten beiden Stücke im 5-Metrum übertragen hat, soll hier der Versuch unternommen werden, auch die ersten fünf Villancicos in diesem Metrum vorzulegen. Anhand einer Abschrift des Cancionero musical de Palacio von der Hand Barbieris, der beiden vorliegenden Übertragungen und der bei Anglés vor den Übertragungen abgedruckten Anfänge der Lieder in der originalen Notation wird also die metrisch-rhythmische Anlage der Villancicos rekonstruiert.

\section{Notenbeispiel 2: Las mis penas, madre (nach Barbieri und nach Anglés)}

Bereits ein Vergleich der ersten Töne zwischen dem Original und den beiden Übertragungen zeigt, daß eine Umschrift, die nicht im 5-Metrum erfolgt, zu Widersprüchen 
führen muß. Die Folge von zwei Semibreves, einer Minima und einer Semibrevis wird von Barbieri bzw. Anglés in folgender Weise wiedergegeben:

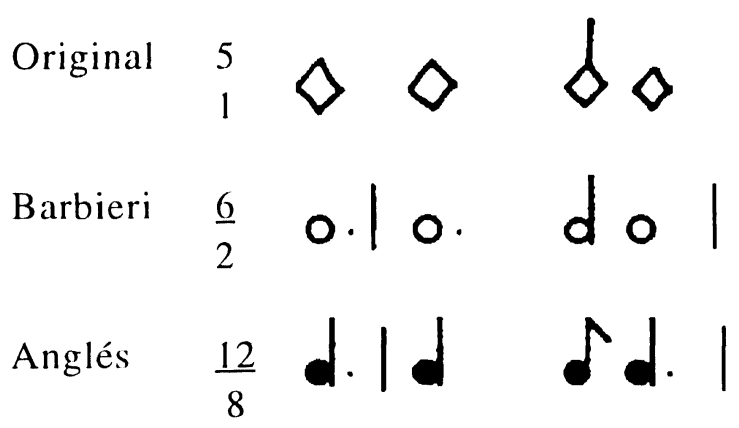

Hält man alle Anfänge der Originalhandschriften nebeneinander, so fällt auf, daß sich auch die Komponisten im frühen 16. Jahrhundert darüber im klaren waren, daß sie mit der Niederschrift dieser Stücke Außergewöhnliches unternahmen. Obwohl es sich um das gleiche metrisch-rhythmische Schema in fast allen Stücken handelt, werden sehr verschiedene Mensurzeichen verwendet.

\section{Notenbeispiel 3: Anfänge der sieben Stücke (Ausgabe Anglés)}

Von zwei Komponisten, Anchieta und Encina, liegen je zwei Stücke vor. Doch nur Anchieta setzt in beiden Fällen dasselbe Zeichen $\mathbf{O}$ (tempus perfectum). Seine Stücke weisen ein höheres inneres Tempo auf als die übrigen, was sich daran zeigt, daß hier doppelt so lange Notenwerte verwendet werden (integer valor $=5$ Minimen) als in den übrigen Stücken (integer valor $=5$ Semibreven). Encina verwendet einmal $\mathbf{C}$ (tempus perfectum diminutum), und im zweiten Fall zeigt er durch die Vorgabe 32, daß er das 5Metrum als Kombination eines Dreier- und eines Zweierabschnittes versteht, was im 16. Jahrhundert einmalig sein dürfte. Die Variationsbreite in der Benennung des ungewöhnlichen Metrums zeigt sich auch darin, daß in einem Fall (Nr. 5) nur im Discantus eine Angabe erscheint und die Notenköpfe geschwärzt, also koloriert wurden, was in den Cancioneros des frühen 16. Jahrhunderts eher selten zu beobachten ist, im vorliegenden Fall aber, da die Minima der kürzeste Notenwert ist, zu keinen Komplikationen führt.

Auffällig ist auch, daß wie bei allen homophonen Sätzen des Cancionero, die nicht auf dem Tactus beginnen, Pausen vorgezeichnet wurden. Zweifellos handelt es sich dabei um eine Hilfe für die Musiker, da ihnen dadurch angezeigt wurde, daß das Stück nicht mit dem Dreierabschnitt eines Tactus, also "abtaktig", sondern "auftaktig" mit dem Zweierabschnitt beginnt.

Daß sich die Stücke auf eine tänzerische Vorlage beziehen, ergibt sich nicht nur aus dem vorzüglich aus der getanzten Musik bekannten Taktmaß von fünf Zeiten pro Einheit, sondern auch aus der Tatsache, daß als rhythmischer Baustein von der Länge 
eines integer valor notarum in allen Stücken folgende Kombination von Notenwerten erkennbar ist:

$$
d_{2}+d_{1}^{\vdots} d
$$

Anglés weist auf den in der spanischen Volksmusik nicht unüblichen 5-Takt hin (s. a. Schindler, Nr. 309, 340, 556, 557). Am bekanntesten ist wohl der baskische Volkstanz Zortcico oder Zorcico mit dem Grundrhythmus

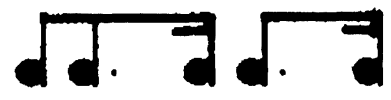

(Crivillé i Bargalló, S. 236). Die in Frage stehenden Villancicos zeigen jedoch eine andere Gliederung $(2+1)$ des ersten der beiden ungleich langen Tactusabschnitte als der Zortcico $(1+2)$. García Matos zeigt in dem Artikel Sobre algunos ritmos de nuestro folklore musical, daß manche Tänze, z. B. die charrada picada aus der Gegend von Salamanca, falsch in 3-, 6oder 9-Takten transkribiert wurden und korrekterweise in einem 10-Takt, der aus zwei Hälften zu je fünf Schlägen besteht, notiert werden müssen. Solch ein asymmetrischer Takt, der besonders deutlich von den Trommelspielern geschlagen wird, kommt dann dem in den Villancicos erkennbaren rhythmischen Muster sehr nahe:

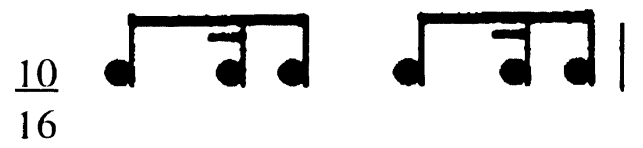

$\mathrm{Da}$ solche Tänze in asymmetrischen Takten aber nicht erst in neuer Zeit existieren, weist die musikethnologische Forschung in der ersten Hälfte unseres Jahrhunderts nach. Noch Donostia zeigt sich 1928 überrascht über zwei gedruckte Zortcicos im 5-Takt aus dem 18. Jahrhundert. Stevenson (1960) zitiert in dem umfangreichen Kapitel über das Cancionero musical de Palacio den spanischen Musiktheoretiker Pedro Salinas, der in seinen De musica libri septem (1577) bereits im 16. Jahrhunderts auf 5-Metren in spanischen Liedern eingeht und deren Ursprung in der Volksmusik sieht. In den Notenbeispielen, die Salinas bringt (S. 272 f), kann man zwei der drei möglichen Kombinationen von zwei längeren Notenwerten (Semibreves) und einem kürzeren (Minima) zu einem Tactus erkennen:

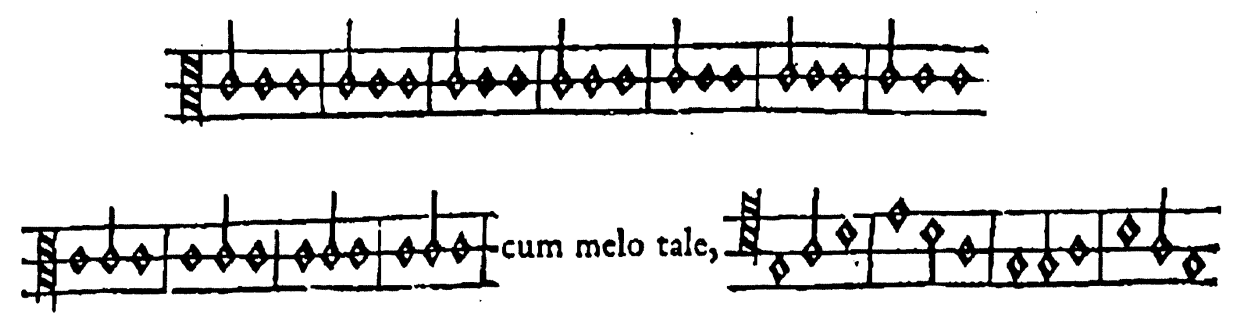


Im Zusammenhang mit diesen Notenbeispielen bemerkt Salinas, leider ohne konkreter zu werden, folgendes:

Cum melo tale, quod ex cantilena quadam Hispanica desumptum est, cuius inter metra fiet mentio. [...]

Et in sonis ex cantilena vulgari Hispanica tale sumi potest.
Solches zeigt eine Melodie, die aus einem gewissen spanischen Lied genommen ist, das unter den Metren erwähnt werden wird. Hinsichtlich der Töne (Tonhöhen) kann solches aus einem spanischen Volkslied genommen werden ( $=$ in einem spanischen Volkslied gefunden werden).

Im ersten der beiden zitierten Sätze wird auf ein weiteres Kapitel verwiesen, und zwar, wie sich zeigt, auf das Kapitel 13 (S. 334 ff) mit der Überschrift De metris, quae fiunt ex pedibus quinque temporum, quae paeonica uno nomine ab his, qui de re metrica scripserunt nuncupantur (Von den Metren, die aus Versfüßen zu fünf Zählzeiten bestehen, welche päonisch von denen genannt werden, die über Metrik geschrieben haben). Unter den verschiedenen spanischen Liedbeispielen, die Salinas bei seiner Gegenüberstellung lateinischer und spanischer Verse zur Erläuterung prosodischer Regeln zitiert, ist erfreulicherweise auch eines der fraglichen Villancicos, und zwar das als letztes aufgeführte Tan buen ganadico (hier phonetisch richtiger als Tam buen ganadico). Zur Veranschaulichung des akatalektischen Trimeter, der aus zwei Bacchei $(\cup--)$ besteht, stellt Salinas das lateinische Amicos vocantes dem spanischen Tam buen ganadico gegenüber (S. 336).

Das Wort-Ton-Verhältnis, wiewohl in den romanischen Sprachen weniger streng geregelt als in den germanischen Sprachen, was den Zusammenfall von Text- und Taktschwerpunkten anbelangt, gibt gerade in tanzähnlicher oder vom Tanz inspirierter Musik wichtige Hinweise auf die Rekonstruktion des musikalischen Verlaufs, da hier schon früh neben der Länge der Noten auch deren Schwere als Ordnungskriterium eingeführt wird. Mit wenigen Ausnahmen sind die Anfangsverse der sieben Villancicos Hexasillabi. Ein Vergleich der Anfänge zeigt eine weitgehende Übereinstimmung des metrischrhythmischen Verlaufs: 


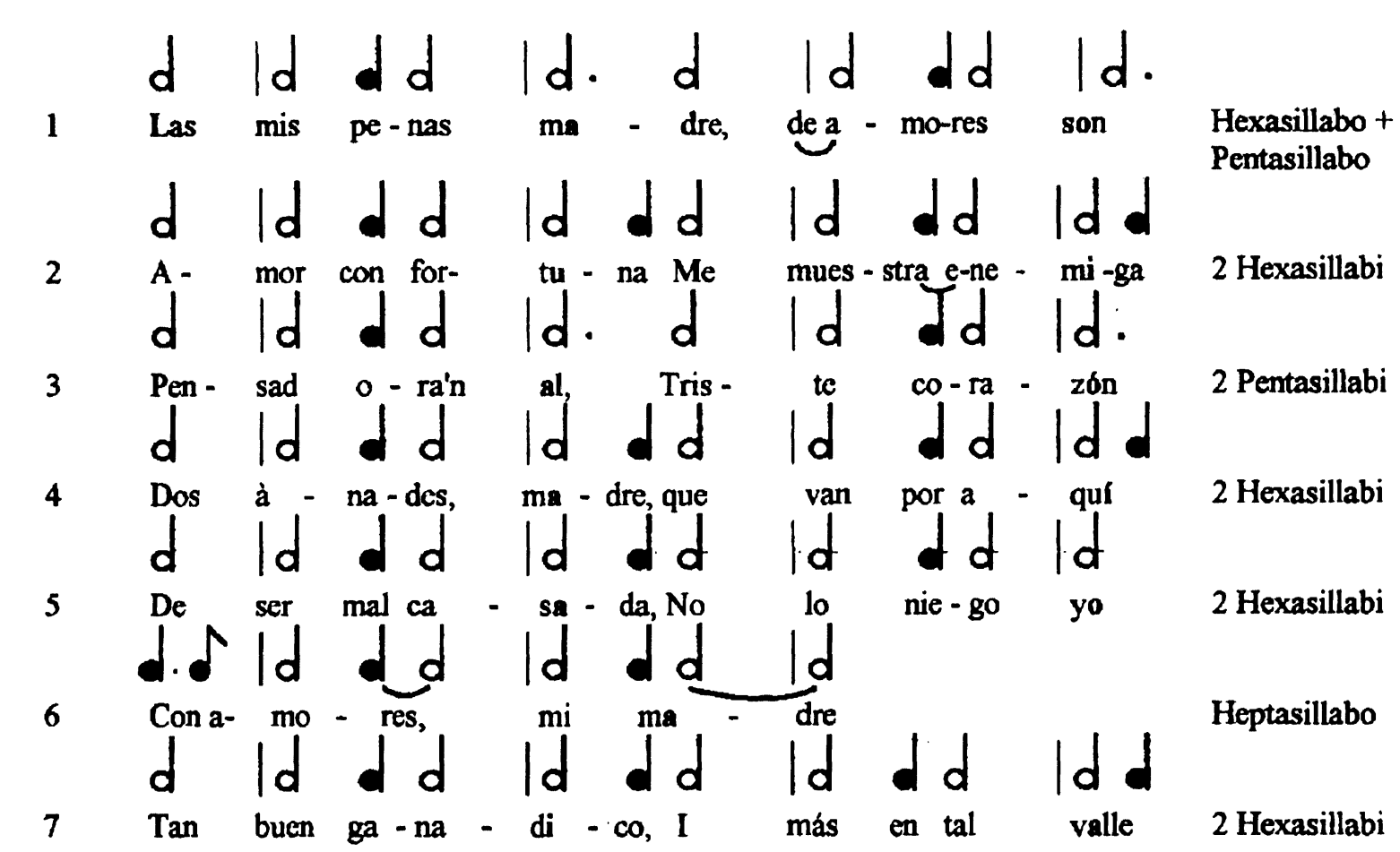

(Die letzten beiden Beispiele wurden des Vergleichs wegen mit Zählzeiten in Halben notiert.)

Die Verse werden also, unbeschadet ob sie paroxytone oder oxytone Endungen aufweisen, in solcher Weise der Musik unterlegt, daß sich Auftakte und dreizeitige Tactuseinheiten ergeben. In den wenigen Fällen, wo Silben fehlen, weil ein Pentasillabo vorliegt, wird durch Dehnungen der regelmäßige Ablauf gewahrt. Ein Ausnahme macht nur das in mehrfacher Hinsicht von den anderen abweichende Villancico 6, da hier ein Heptasillabo und ein eher melismatisches Wort-Ton-Verhältnis vorliegen.

Schwierigkeiten ergeben sich bei den Zäsuren an den Strophengrenzen. Zu Beginn der beiden musikalischen Abschnitte, in die ein Villancico zerfällt, am Ende von Estribillo und Copla also, ist meist die gleiche Folge von Pausen verzeichnet. Am Ende der Copla liegen außerdem jeweils die gleichen langen Notenwerte vor wie am Beginn des Estribillo, nämlich Longae. Diese langen Schlußklänge und jene Binnenpausen lassen den musikalischen Ablauf fast zum Stillstand kommen, wenn man sie wörtlich nimmt. Der Gedanke liegt nun nahe, den Übergang von einem Abschnitt zum anderen oder zum selben Abschnitt, wenn es sich um eine Wiederholung handelt, in gleicher Weise zu raffen. Dazu muß der Schlußton eines Abschnitts so weit verkürzt werden, daß er mit dem auftaktigen Beginn des folgenden Abschnitts einen vollständigen Tactus ergibt. Nur auf diese Weise vermeidet man, daß der tanzartige Duktus der Stiicke unterbrochen wird. 
Die beiden Möglichkeiten seien am Discantus des Villancicos Las mis penas, madre einander gegenübergestellt. Im zweiten Vorschlag entfällt ein ganzer Tactus. Anglés deutet eine derartige Lösung übrigens im sechsten Villancico (Con amores, mi madre) an, wenn er die im Manuskript enthaltenen Pausen, wie aus der Fußnote ersichtlich, in eckige Klammern setzt.

\section{Notenbeispiel 4: Discantus des Villancicos Las mis penas, madre in zwei unterschiedlichen Übertragungsformen}

Bei der zweiten Lesart der einzelnen musikalischen Abschnitte, genauer: ihrer Anfänge und Schlüsse, ergibt sich in den meisten Fällen eine gerade Taktanzahl dieser Abschnitte:

Nr. 1 Las mis penas, madre, De amores son.

$4+2$

Salid, mi señora,

Nr. 2 Amor con fortuna Me muestra enemiga. No sé que me diga.

No sé lo que quiero, Pues busqué mi daño;

Nr. 3 Pensad ora'n al, Triste corazón, Pensad ora'n al Qu'en amores non. Pensad en vivir, Contino pecado,

Nr. 4 Dos ánades, madre, Que van por aquí Mal penan a mí.

Dos ánades, madre, Del campo.

Nr. 5 De ser mal casada No lo niego yo, Cativo se vea Quien me cativo.

Cativo se vea $Y$ sin redención;

Nr. 6 Con amores, la mi madre, con amores m'adormí, Con amores m'adormí Así dormida soñava

Nr. 7 Tan buen ganadico, I más en tal valle, Plaser es guardalle.

Ganado d'altura Y más de tal casta,
3

$4+2$

4

$4+4$

4

$4+4$

4

$4+4$

4

$3+3+3$

3

$4+2$

4

Es stellt sich nun die Aufgabe, den Begriff Tactus für die vorliegenden Stücke neu zu definieren. In der Musiktheorie des 16. Jahrhunderts stellt er eine Maßeinheit dar, welche die Aufwärts- und die Abwärtsbewegung der Dirigierfigur umfaßt. Im tactus aequalis, der zweizeitigen Einheit, war die zeitliche Ausdehnung der beiden Bewegungen identisch. Im tactus inaequalis, der dreizeitigen Einheit, umfaßte die Aufwärtsbewegung zwei Zeiten und die Abwärtsbewegung eine. Auch in einem 5-Metrum ist eine Untergliederung des integer valor notarum zu erwarten, eine Nachzeichung aller fünf Einzelglieder bei dem relativ hohen Tempo der tanzähnlichen Sätze aber unwahrscheinlich. Es bleibt daher nur eine Lösung: Pro Tactus hat man mit zwei Pulsen zu rechnen, die ungleich lange Dauern einleiten, und zwar im Verhältnis $3: 2$. Salinas schreibt in diesem Sinne auf Seite 335: ... ut tria tempora in arsi, in thesi duo sint (..., so daß drei Zählzeiten auf der Arsis, auf der Thesis zwei Zählzeiten sind). Ein solcher tactus inaequalis wird auch von dem Mensurzeichen (32) des zuletzt aufgeführten Villancicos Tan buen ganadico nahegelegt. 
Derartige asymmetrische Taktarten sind uns aus der Volksmusik des Balkans bekannt, und sie werden in der Notierung meistens nach Maßgabe des größten gemeinsamen Teilers bezeichnet, so daß eine rationalisierende Taktvorgabe $(5 / 8,7 / 8)$ zur Anwendung kommt, die aber der erklingenden Realität nicht gerecht wird. Bartok notiert in Sechs Tänze in bulgarischen Rhythmen aus dem Mikrokosmos Band 6 sinnvollerweise im Zähler einen Additionsterm, der die ungleichgewichtigen Hauptschläge erst sichtbar macht. Selbst scheinbar symmetrische Taktarten zeigen auf diese Weise ihr wahres Gesicht:

$\begin{array}{lccc}\text { Tanz } & \text { Bartoks Taktangabe } & \begin{array}{c}\text { Taktart mit summiertem } \\ \text { Zähler }\end{array} & \begin{array}{c}\text { Zahl der (ungleich } \\ \text { langen) Hauptschläge }\end{array} \\ 148 & \frac{4+2+3}{8} & 9 / 8 & 3 \\ 149 & \frac{2+2+3}{8} & 5 / 8 & 2 \\ 150 & \frac{5 / 8}{8} & 5 / 8 & 3 \\ 151 & \frac{3+2+3}{8} & 8 / 8 & 4 \\ 152 & \frac{2+2+2+3}{8} & 9 / 8 & 3 \\ 153 & \frac{3+3+2}{8} & 8 / 8 & 3\end{array}$

In allen genannten Fällen ist die Schlageinheit also nicht die Achtel, sondern es liegen ungleich lange - übrigens den Tanzschritten korrespondierende - Einheiten vor. Ein Takt mit der Vorzeichnung $3+2$ ist also ein Zweiertakt mit einer ersten Schlagzeit, die um die Hälfte länger ist als die zweite. Die Taktangabe $2+2+2+3$ meint demnach keinen 9-Takt, sondern einen 4-Takt mit einer gelängten letzten Zählzeit. Ähnliches dürfte auch bei den Villancicos vorliegen: Es handelt sich um 2-Metren mit ungleich langen Zählzeiten: $3+2$.

Die rhythmisch-metrischen Gestalten der sieben Villancicos in der Proportio quintupla sind also weder so unterschiedlich, wie es die Mensurzeichen in der Handschrift suggerieren, noch so verschieden, wie es die Übertragungen nahelegen. Das ungewöhnliche Metrum provozierte verschiedene Versuche der notenschriftlichen Fixierung und in der Folge auch der Transkribierung in moderne Notation.

Interessant bleiben die sieben Villancicos und ihre Transkriptionen aus mehreren Gründen: Sie zeigen einmal mehr die Interdependenz von Volks- und Kunstmusik und sind ein Beispiel dafür, daß Wissenschaft in ihrer Zeit befangen ist und nicht das Unmögliche denken kann. Erst die Erkenntnis, daß asymmetrische Taktarten in der Volksmusik weit- 
verbreitet sind, öffnete Anglés den Blick für das Ungewöhnliche ihrer metrisch-rhyth mischen Gestalt. Die sieben Liedsätze gehören aber nicht nur auf Grund ihres in der Tat überraschenden Metrums zu den reizvollsten Kompositionen des Cancionero Musical de Palacio.

Notenbeispiel 5: Neuübertragung der Villancicos 1 - 5

\section{Ausgaben}

Barbieri, Fr. A.(Hg.): Cancionero Musical de los siglos XV y XVI, Madrid 1890 Anglés, Higinio (Hg.): Cancionero Musical de Palacio (Siglos XV - XVI), 2 Bde., (= La Música en la Corte de los Reyes Católicos, II: Polifonía profana), Barcelona 1947

\section{Literaturnachweise}

Apel, Willi: Die Notation der polyphonen Musik 900 - 1600, Leibzig 1970

Crivillé i Bargalló, Josep: El folklore musical (= Historia de la música española, Bd.7), Madrid 1983

Donostia, José Antonio de: Más sobre la escritura del zortziko en 5/8, in: Revue Internationale des Etudes Basques 1935, S. 331

Donostia, José Antonio de: Dos zortzikos del siglo 18 en $5 / 8$, in: Revue Internationale des Etudes Basques 1928, S. 333

Figueras, José Romeu: Cancionero Musical de palacio, Edición crítica de los textos, 2 Bde.

(= La Música en la Corte de los Reyes Católicos, IV - 2), Barcelona 1965

Ganassi, Sylvestro: La Fontegara, Venedig 1535, hg. von Hildemarie Peter, Berlin 1956. García-Matos, Manuel: Sobre algunos ritmos de nuestro folklore musical, in: Anuario musical 15 (1960), S. 101 und Anuario musical 16 (1961), S. 27

Geiger, A.: Bausteine zur Geschichte des iberischen Vulgär-Villancico, in: ZfMw IV, 1921/22, S. 65

Pope, Isabel: Musical and Metrical Form of the Villancico, in AnnMl II, 1954, S. 189

Salinas, Francisco: De Musica, Salamanca 1577 (Faksimile-Nachdruck, Kassel und Basel 1958)

Stevenson, Robert: Spanish Music in the Age of Columbus, Den Haag 1960 
Notenbeispiel 1

335. Con amores, mi madre

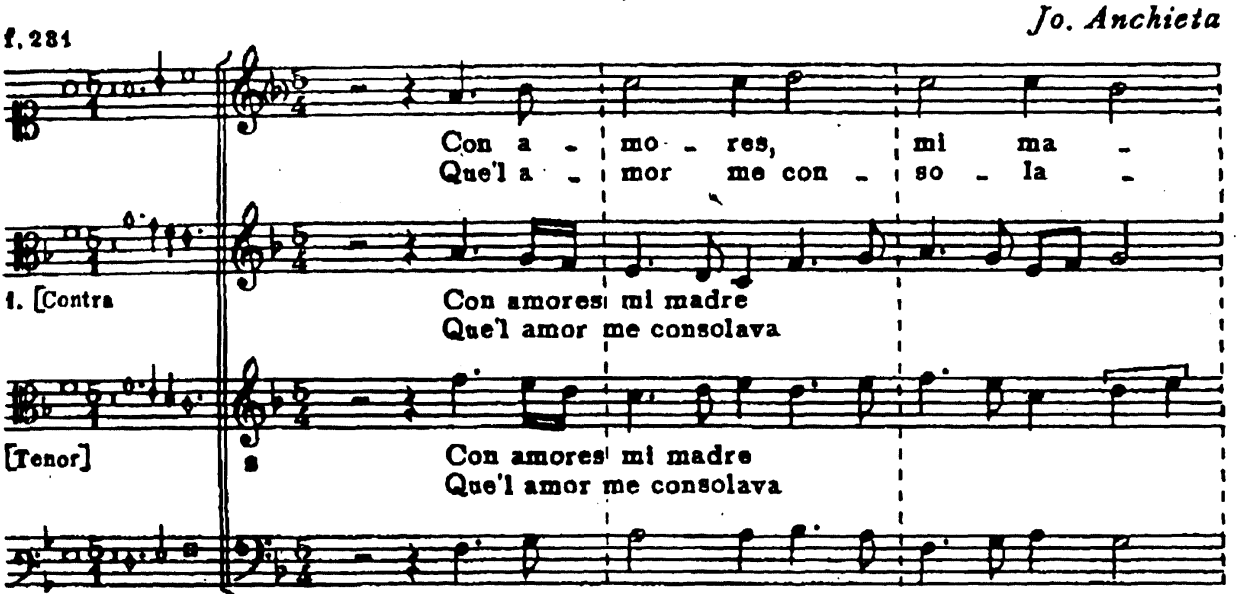

Con amores mi madre

Que'l amor me consolava
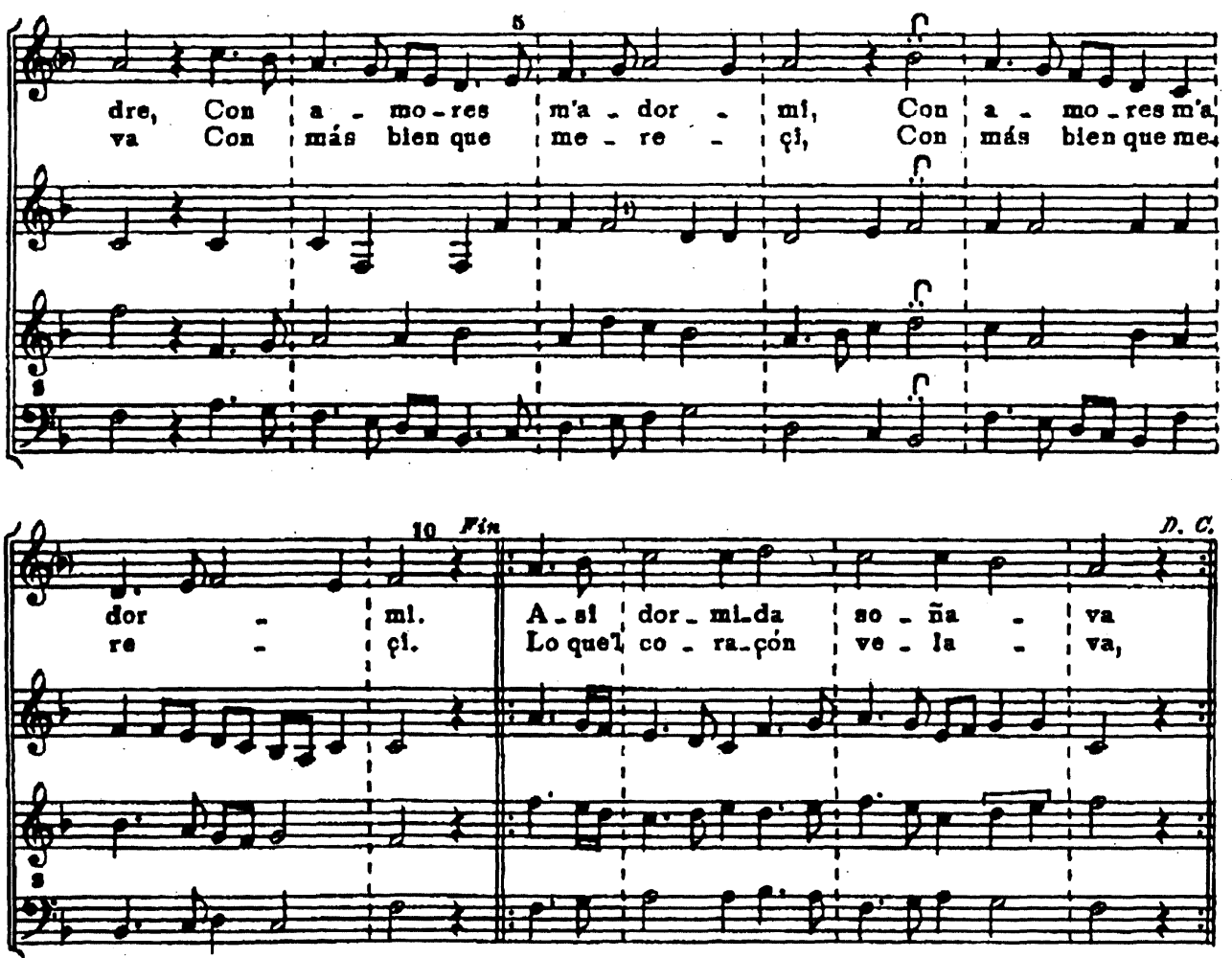

Adormeçlome ol favor

Qu'amor me dló con amor;

Dló deacanso a mi dolor

La fo con que le servi.

1) Ma. sobra yn fao

Inotituto Bupestal de Mualcolorla 
426. Tan buen ganadico
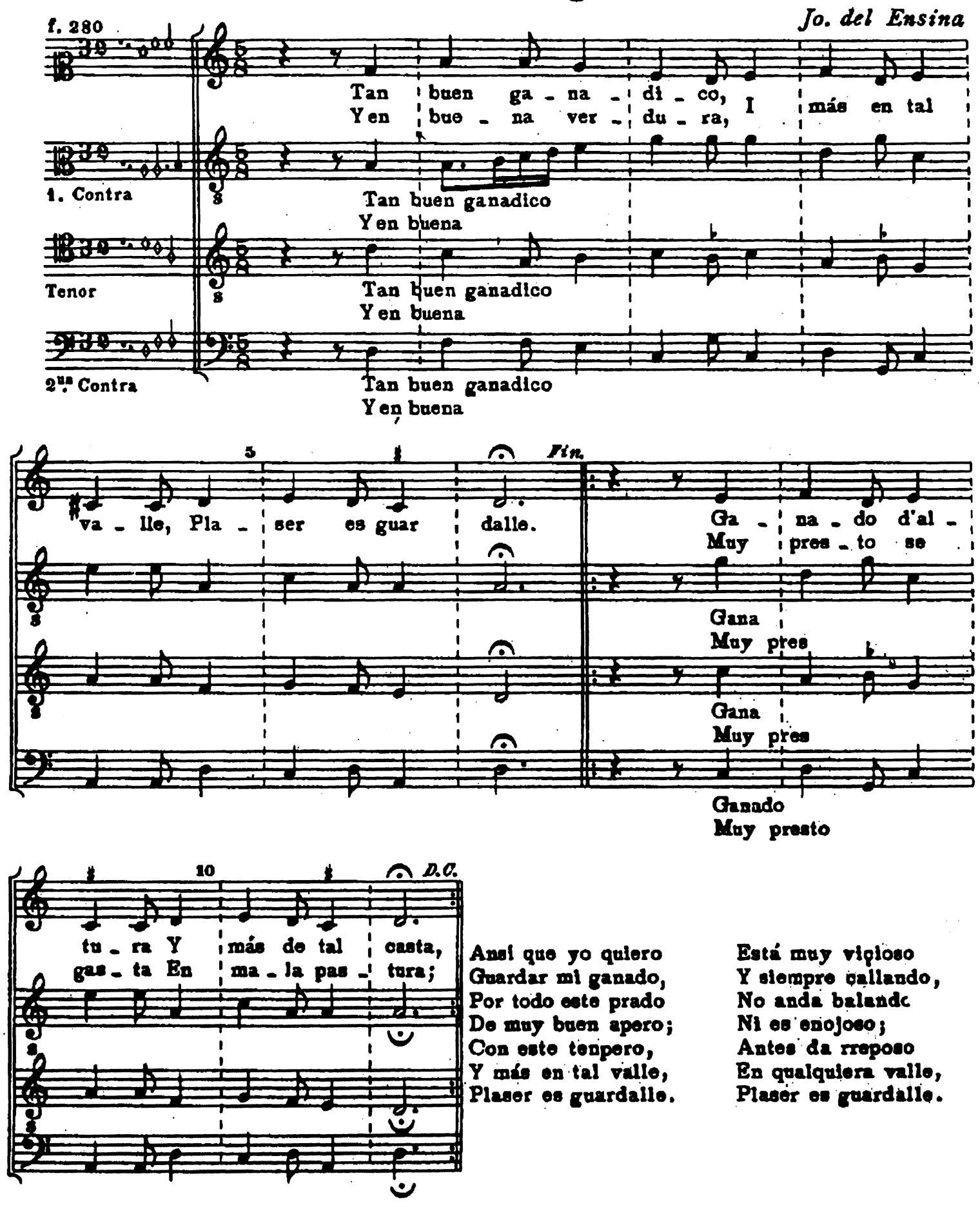

And que jo quiero

Guardar mi ganado,

Por todo ceto prado

Do muy buen aporo;

Con este teriporo,

$Y$ mís on tal villo,

Pleser os guardallo.
Está muy viploeo

$Y$ slempro oallardo,

No ande balende

Ni es eriojoeo;

Anter da sraposo

En qualqulere vallo, Placer es crardalie. 
Notenbeispiel 2
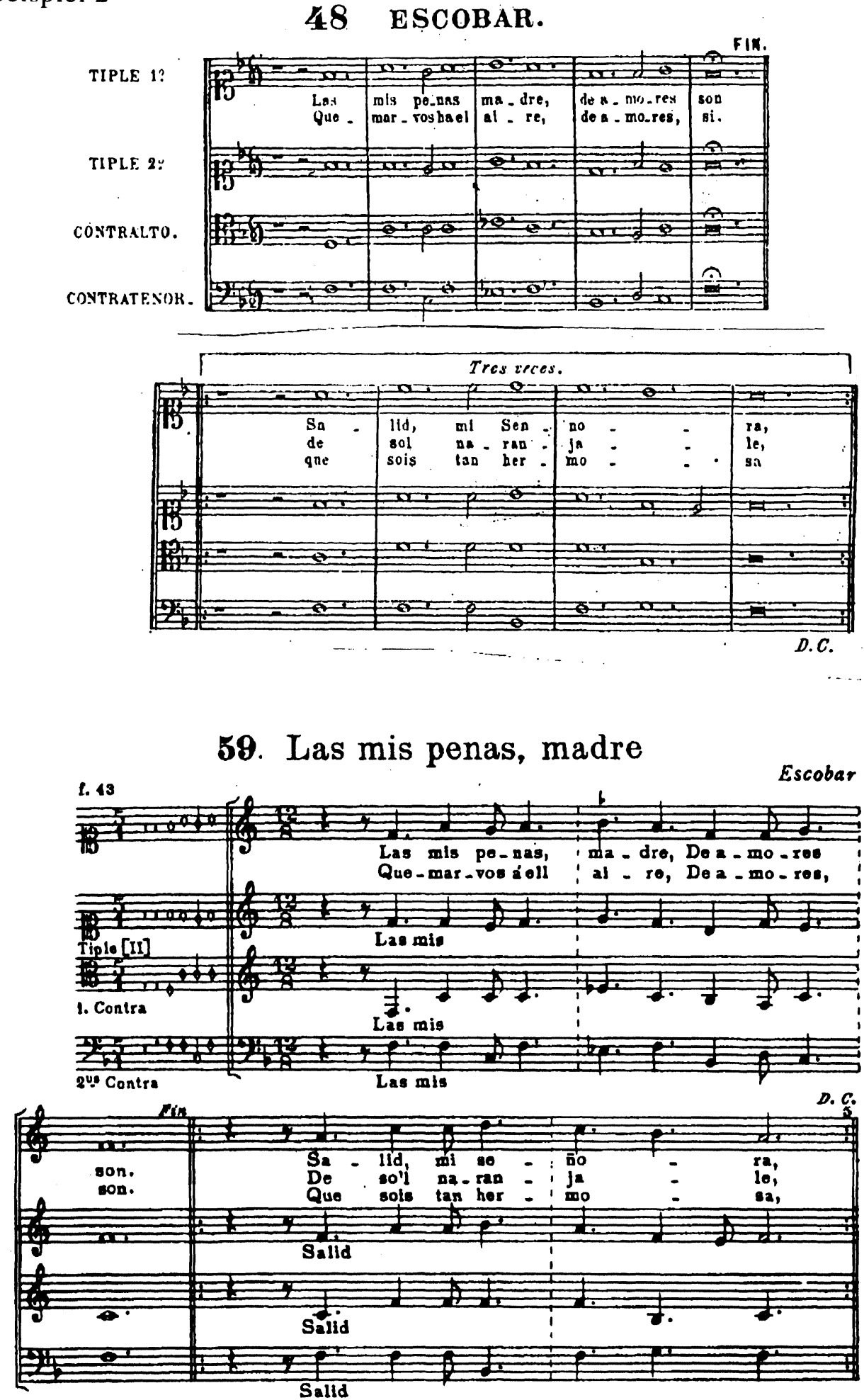

[12] 
Notenbeispiel 3
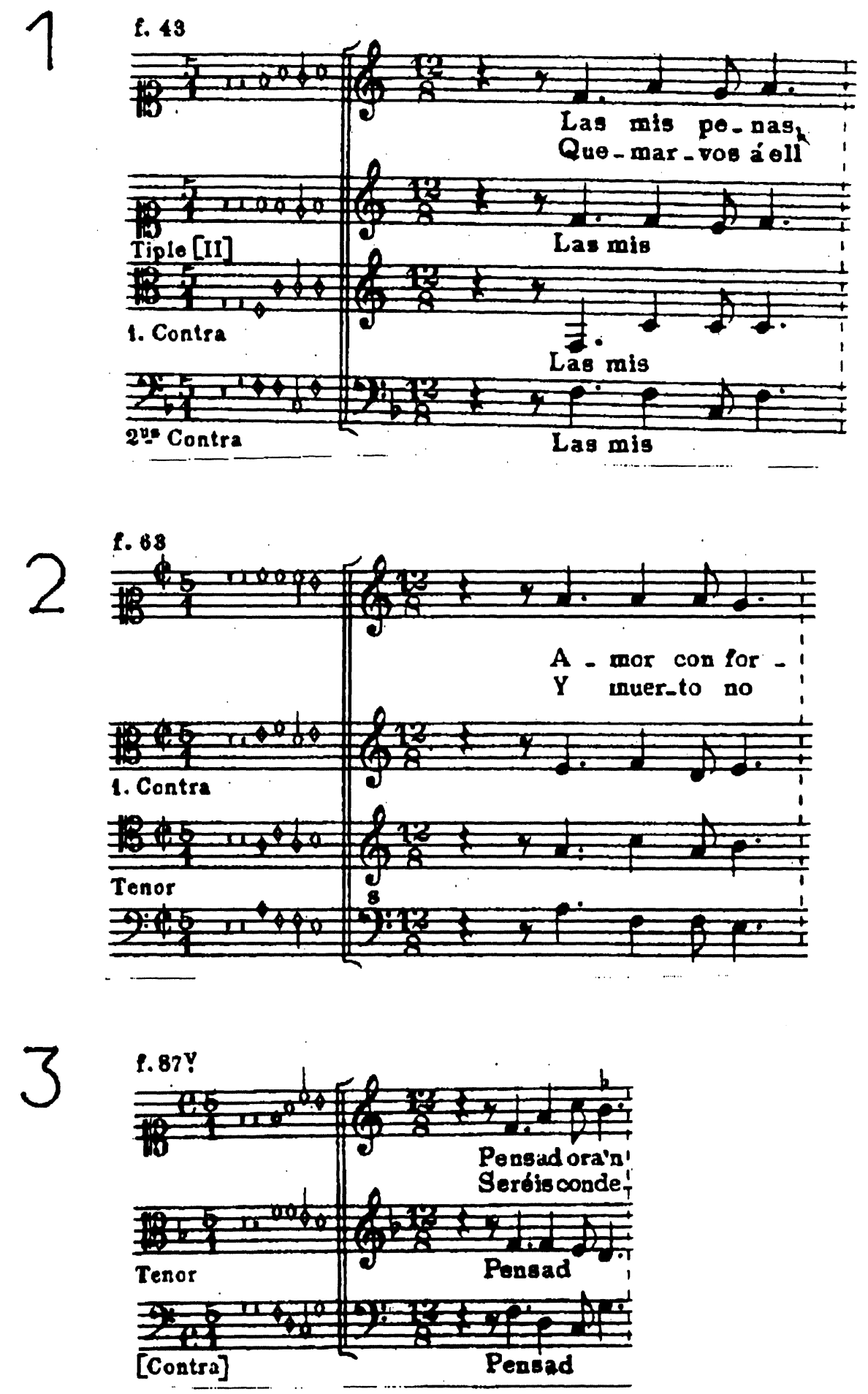

[13] 

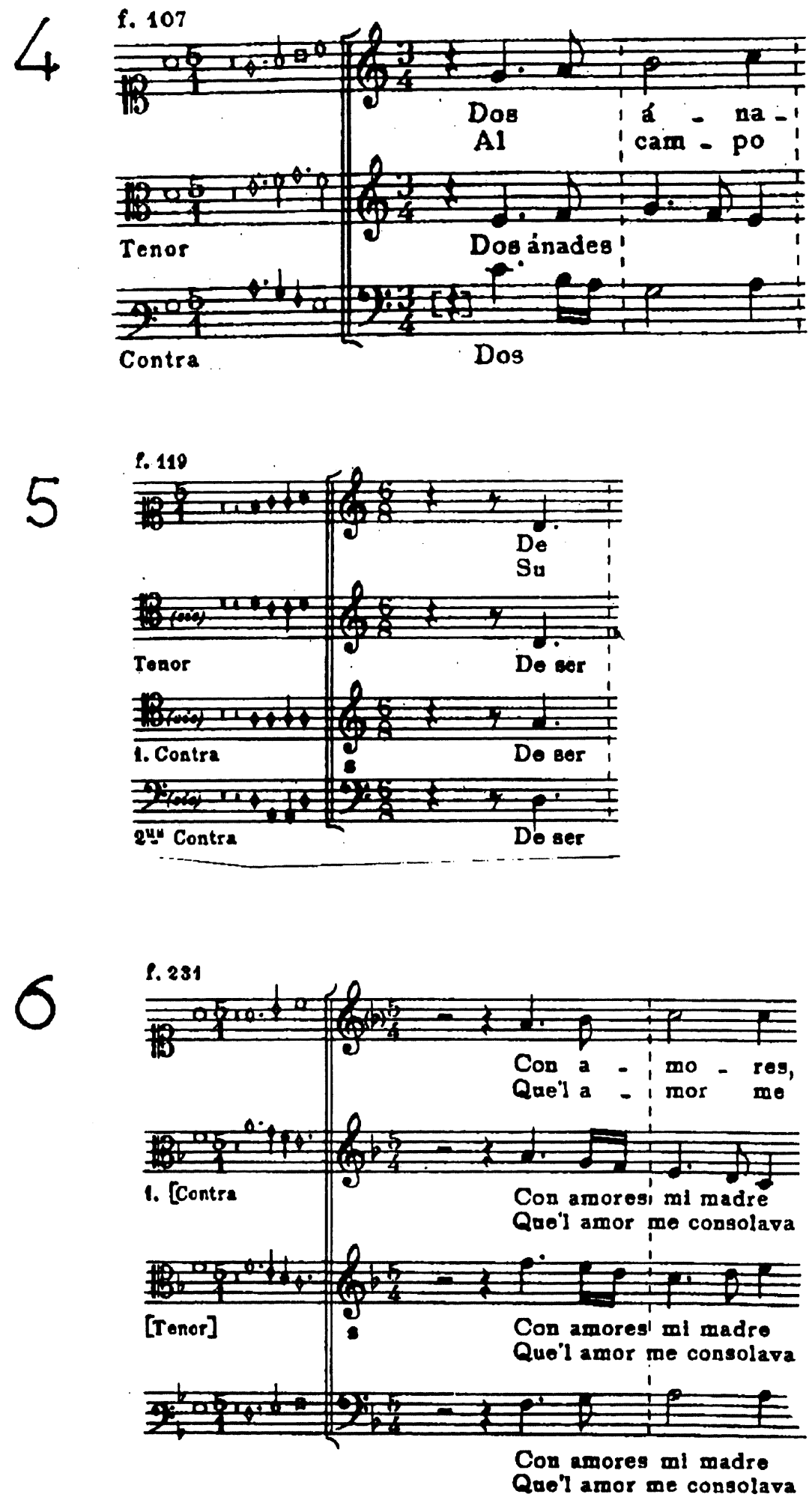
7

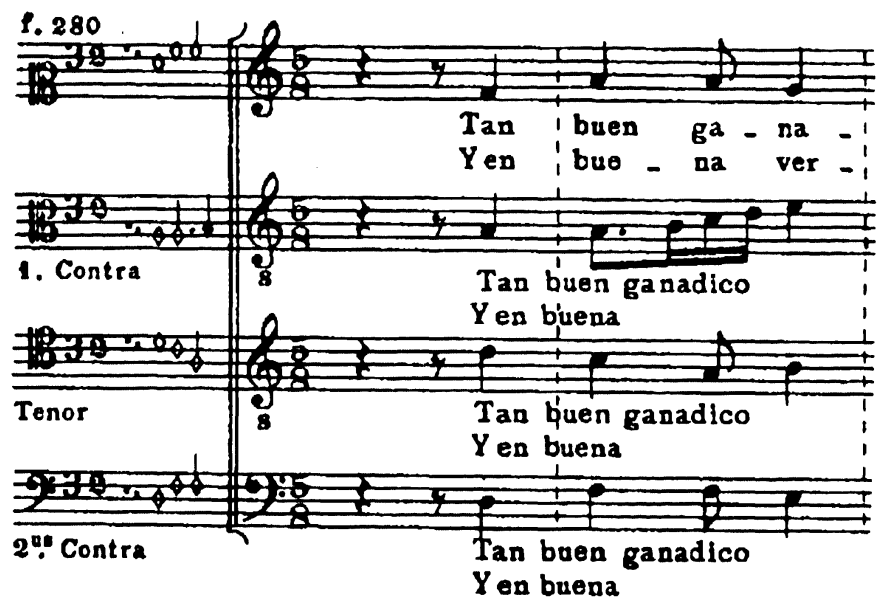

Notenbeispiel 4
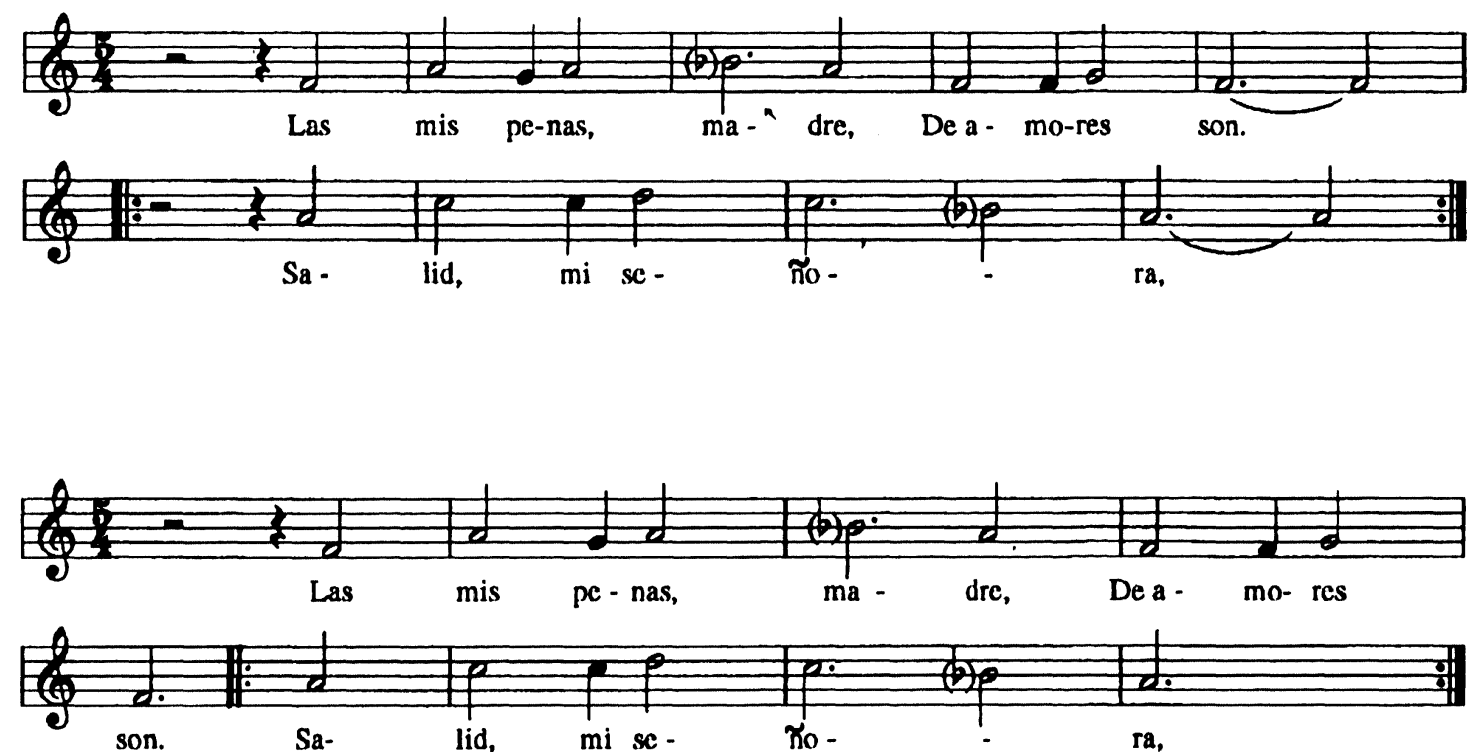
Notenbeispiel 5

\section{Las mis penas, madre}

Escobar
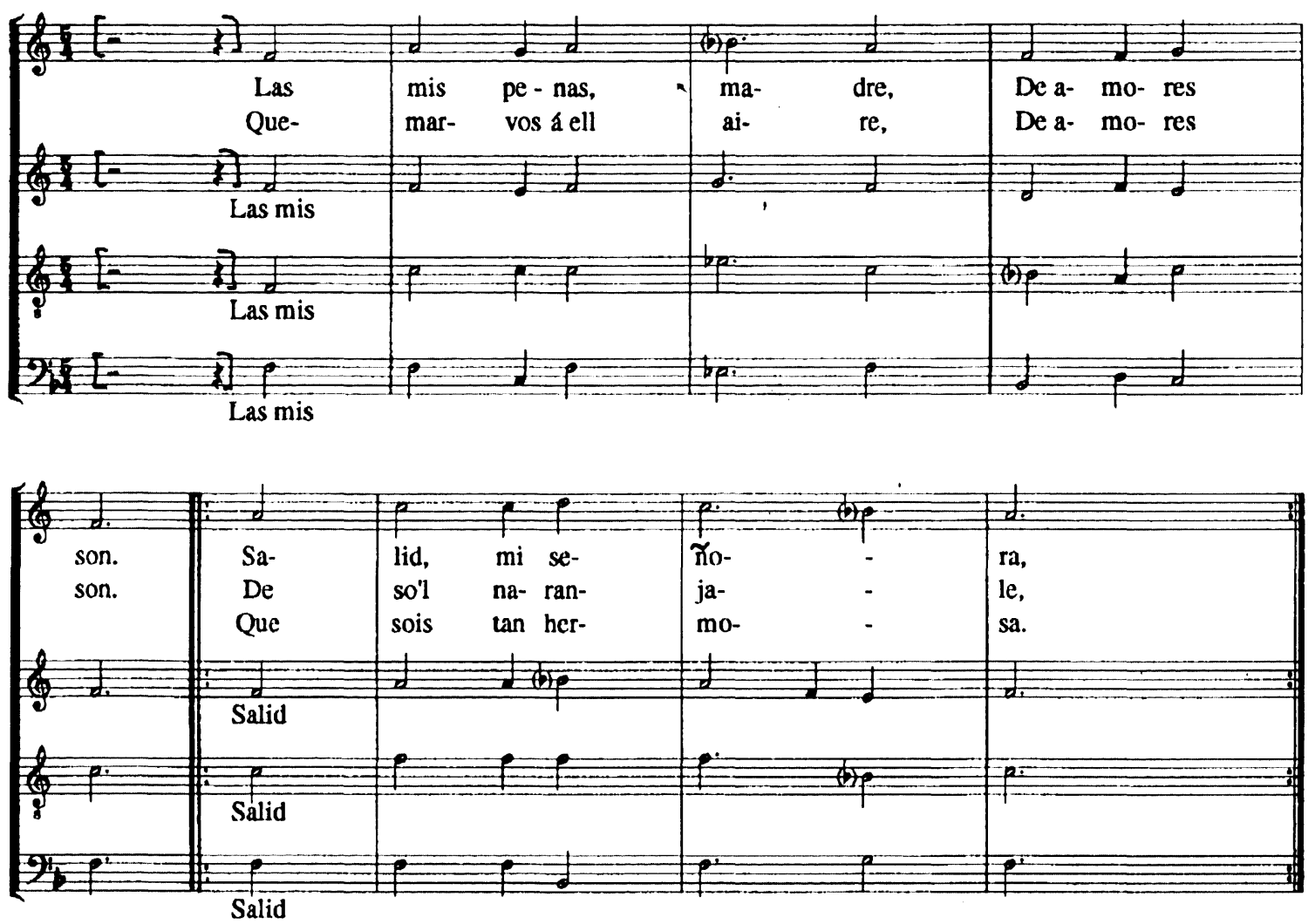

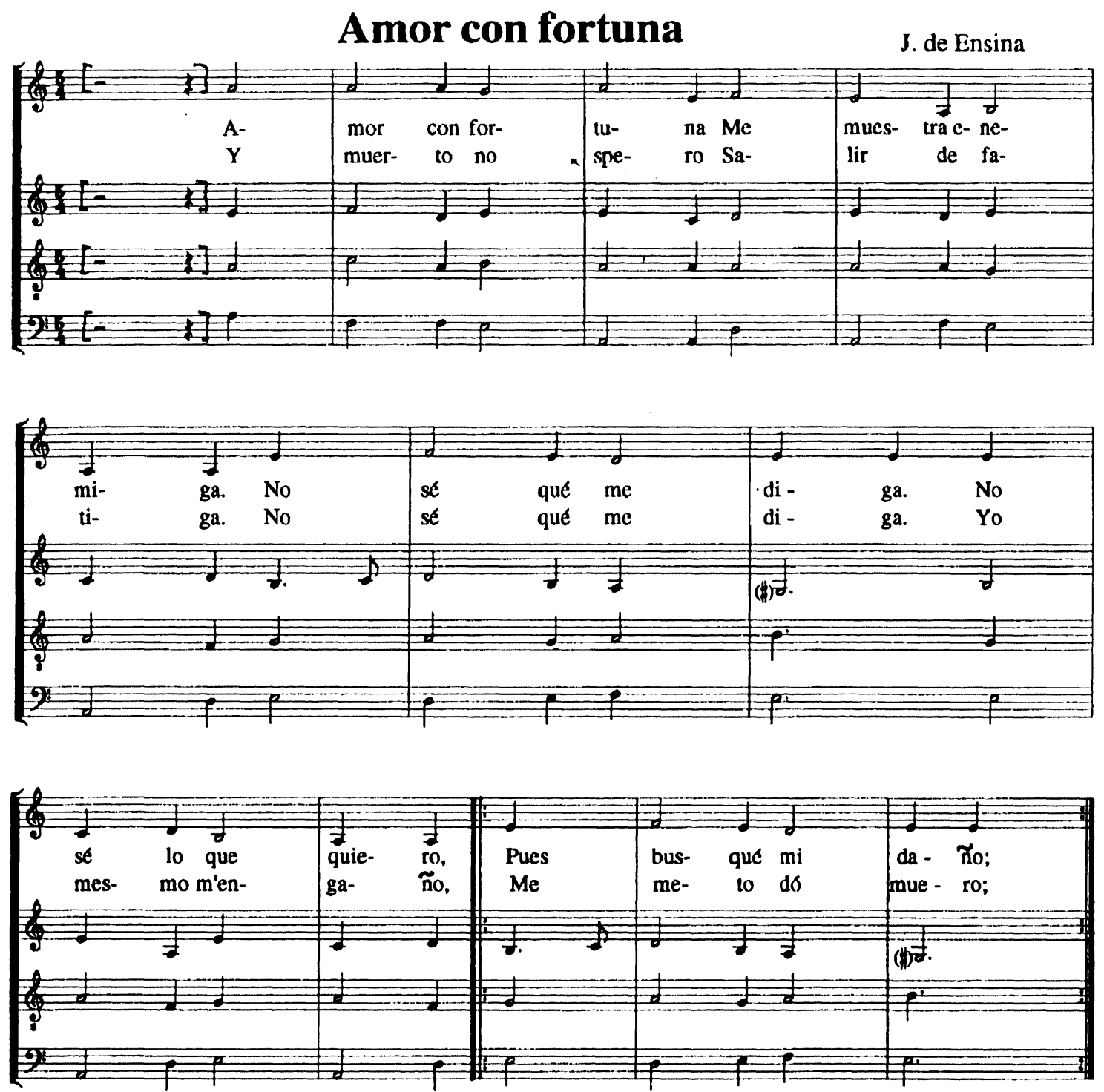
Pensad ora'n al
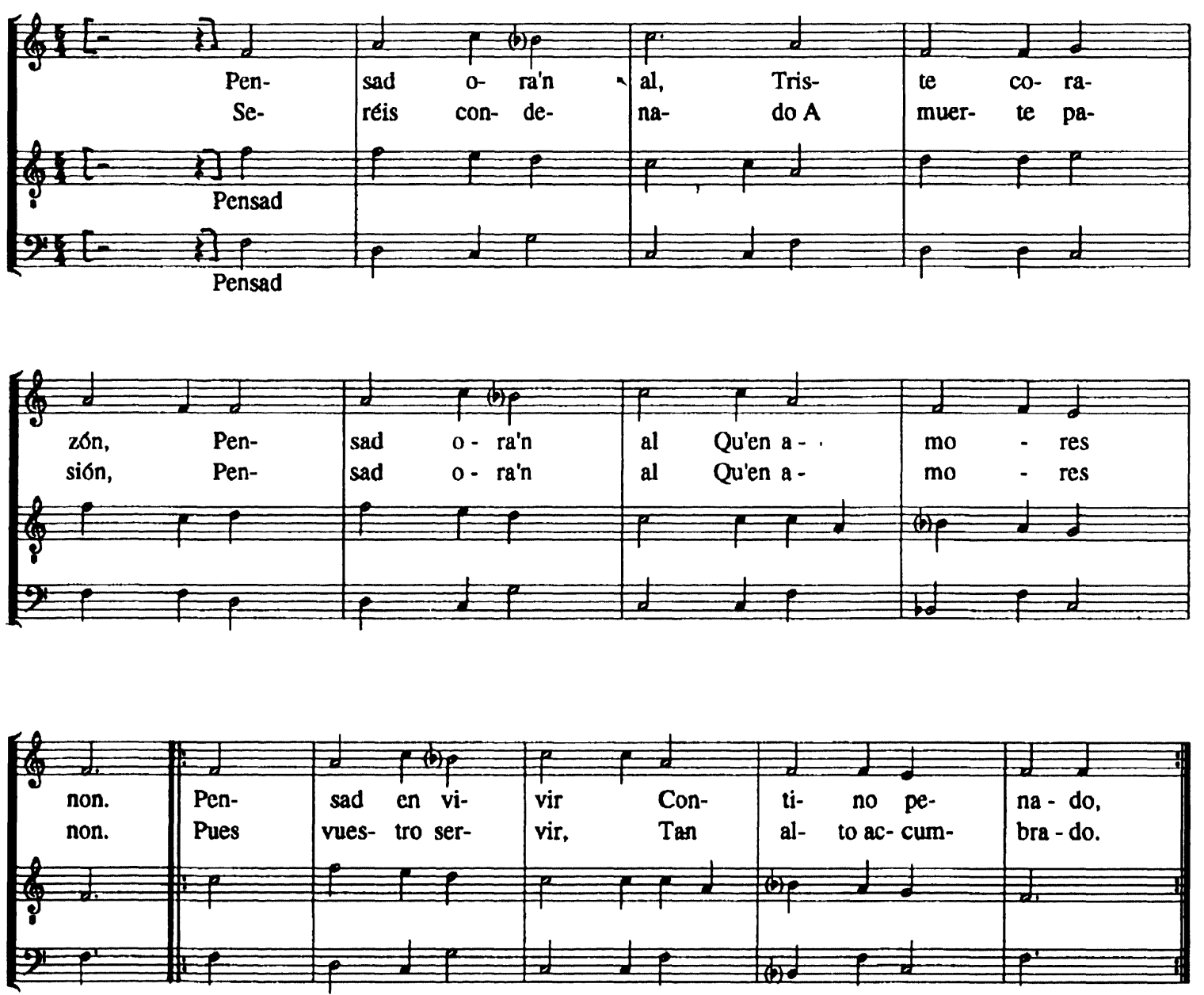


\section{Dos ánades, madre}
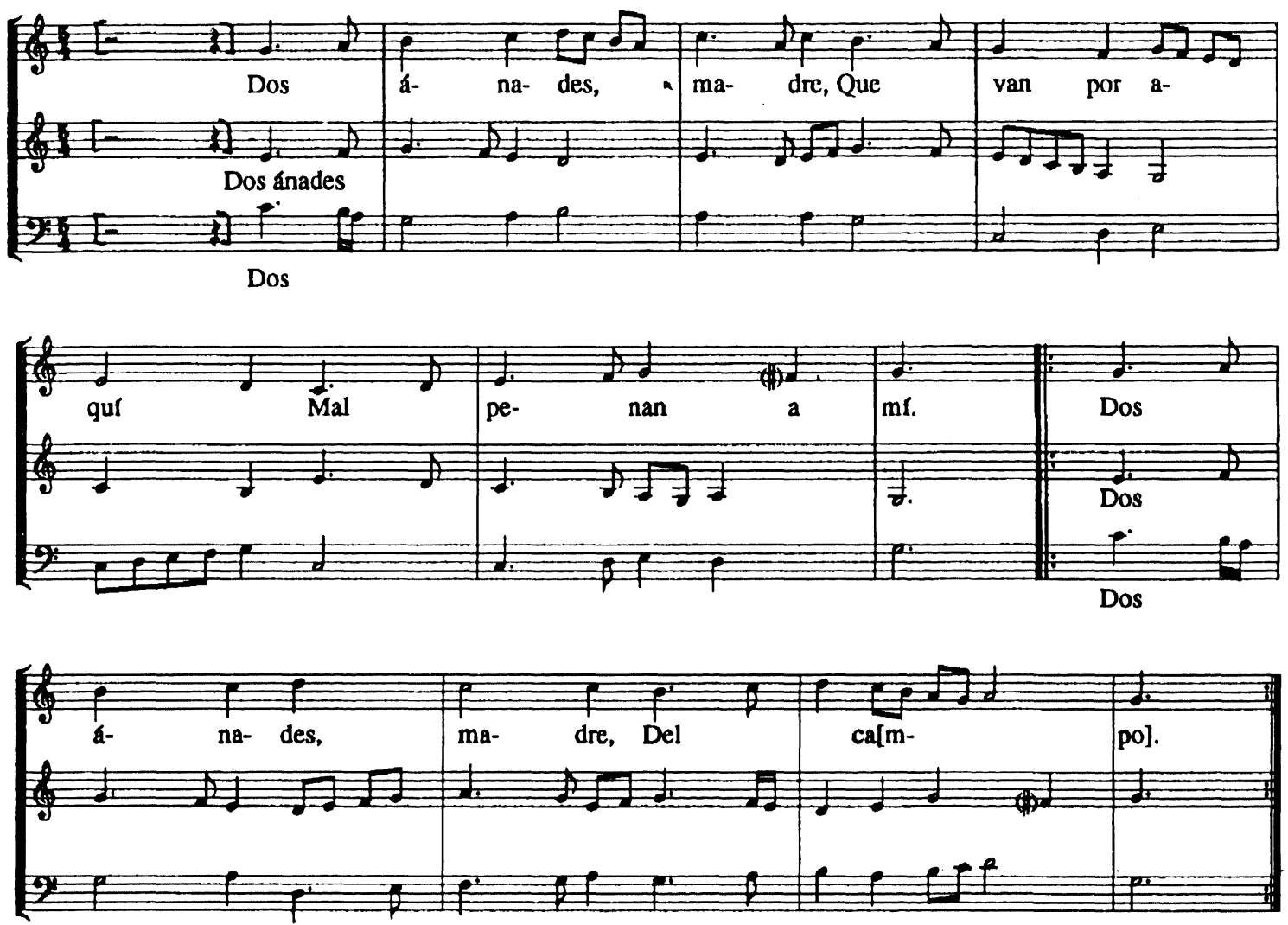


\section{De ser mal casada}

Do. Fernandes
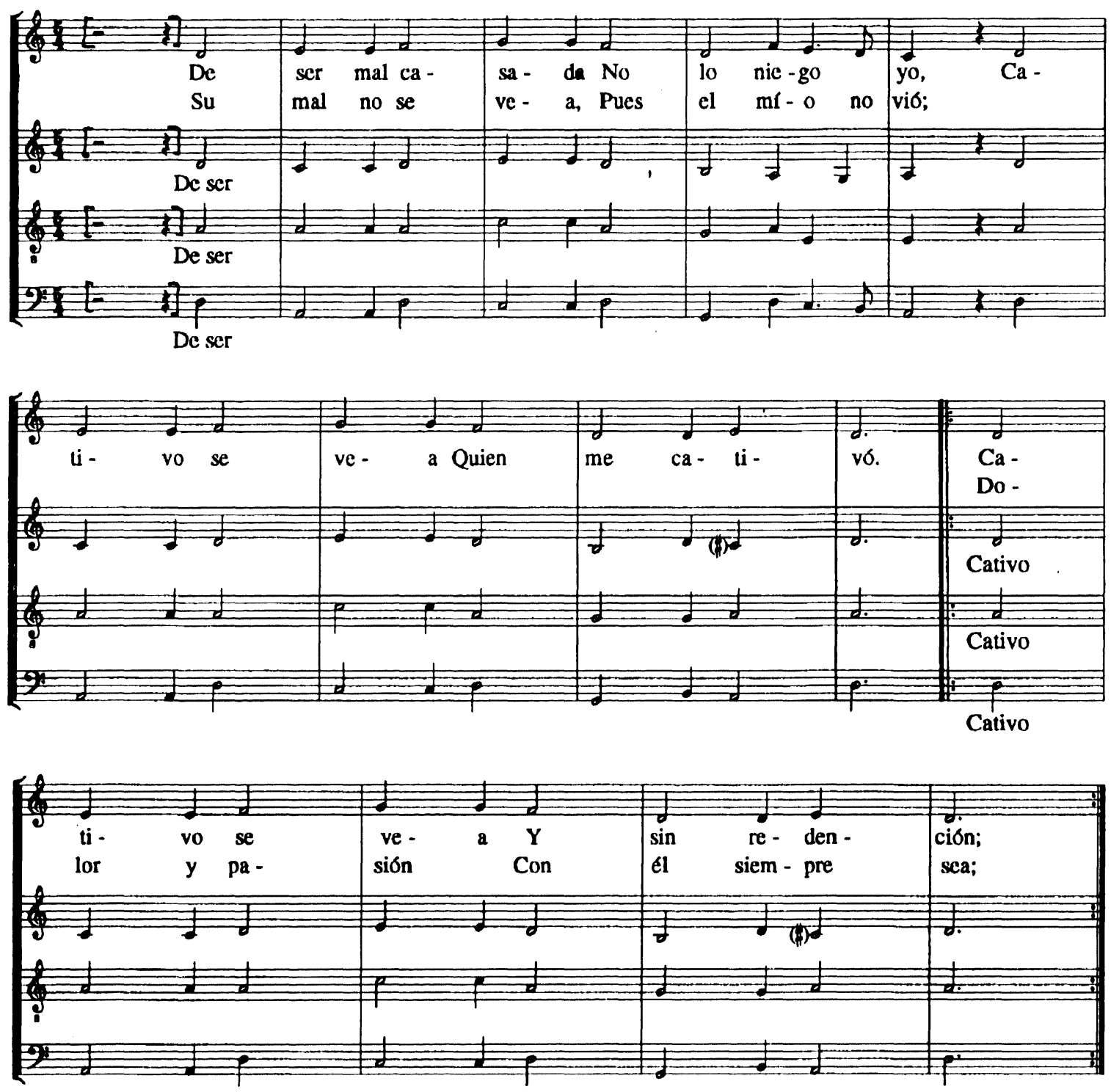